\title{
METODOLOGÍA DIAGNÓSTICA ANTE LA RECIDIVA BIOQUÍMICA DESPUÉS DE BRAQUITERAPIA.
}

\author{
José F. Suárez Novo, Sergi Pastor López, Fernando Aguiló Lucia y Eladio Franco Miranda.
}

Servicio de Urología. Hospital Universitario de Bellvitge. L' Hospitalet de Llobregat. Barcelona. España.

\begin{abstract}
Resumen.- En la actualidad el cáncer de próstata es la neoplasia no cutánea más frecuente entre los hombres en USA, ocupando en nuestro país la segunda posición tras el cáncer de pulmón. En los últimos años se ha modificado el perfil del cáncer de próstata diagnosticado debido a la amplia difusión de la determinación del PSA. En la actualidad aproximadamente el $47 \%$ de los cánceres de próstata que se diagnostican son de bajo riesgo. En esta situación terapias mínimamente invasivas como la braquiterapia cada vez tienen una mayor aceptación en nuestro medio. Se analiza la especial cinética del PSA tras la braquiterapia y la dificultad que entraña el diagnóstico de la recidiva bioquímica tras braquiterapia realizando una revisión de la evidencia científica disponible en la literatura.
\end{abstract}

Palabras clave: Cáncer de próstata. Braquiterapia. Recidiva bioquímica. PSA bounce.

Summary.- Currently prostate cancer is the most frequent extracutaneous neoplasia in males in the USA, and second after lung cancer in our country. Over the last years the profile of prostate cancers diagnosed has changed due to the wide diffusion of PSA determination. Currently, almost $47 \%$ of prostate cancers are low risk at diagnosis. In this situation, the minimally invasive therapies such as brachytherapy have a growing acceptance in our environment. We analyze the special PSA kinetics after brachytherapy, and the difficulty entailed by the diagnosis of biochemical recurrence after brachytherapy, performing a bibliographic review of the available scientific evidence.

Keywords: Prostate cancer. Brachytherapy. Biochemical recurrence. PSA.

\section{INTRODUCCIÓN}

El cáncer de próstata es ya la neoplasia no cutánea más frecuente entre los hombres en los EE.UU., siendo estimados unos 232.000 nuevos casos en el 2005, con una mortalidad relacionada con la enfermedad de unos 30.350 pacientes en dicho periodo (1). En el Estado Español el cáncer de próstata se sitúa en el segundo o tercer puesto de las neoplasias más prevalentes entre los varones, por detrás del cáncer de pulmón y, en algunos registros de tumores, por detrás del colorectal (2). 
La amplia difusión en la utilización del antígeno prostático específico, ya sea dentro de campañas de screening 0 al margen de las mismas, ha implicado un cambio en las características del cáncer de próstata que se diagnostica en la actualidad. Según el registro de CaPSURE y utilizando los criterios de riesgo propuestos por D'Amico (3), se ha pasado de un porcentaje del $30 \%$ en el periodo 1989-1990 a uno del $47 \%$ en el periodo $2001-2002$ de pacientes afectos de carcinoma de próstata de bajo riesgo. El riesgo intermedio ha pasado de un $34 \%$ en el 1989 1990 a un $37 \%$ en el 2001-2002, mientras que el alto riesgo ha disminuido dramáticamente, pasando de un $36 \%$ en los años $1989-1990$ a un $16 \%$ en el periodo 2001-2002 (4).

La migración en los grupos de riesgo hacia tumores menos agresivos, el mejor conocimiento de la historia natural de esta patología y una mayor consciencia en cuanto a los cambios en la calidad de vida que los tratamientos implican han variado la tendencia en cuanto al tratamiento primario aplicado a los pacientes. El incremento en el porcentaje de pacientes diagnosticados con tumores de bajo riesgo junto con la comunicación de unos excelentes resultados en cuanto a supervivencia libre de enfermedad bioquímica $(5,6)$ han hecho que la braquiterapia pase a ser el tratamiento realizado en un $5.7 \%$ de los pacientes en el periodo 1996-1998 a un $18.4 \%$ en el 1999-2001; durante el mismo lapso de tiempo la prostatectomía radical ha pasado de un $54.9 \%$ a un $51.9 \%$, mientras que la radioterapia externa pasa de un $10 \%$ a un $6.8 \%$ (7).

Este incremento en los pacientes tratados con braquiterapia plantea toda una serie de nuevos escenarios desconocidos hasta el momento. La peculiar cinética del PSA post-braquiterapia, la ausencia de un criterio claro para medir la recidiva bioquímica tras la misma, la ausencia de una pauta estándar de diagnóstico de ésta recidiva y la aún menos protocolizada pauta de rescate terapéutico, plantean todo un reto al clínico ante una situación, que si bien infrecuente, no es del todo excepcional en la práctica diaria.

\section{CINÉTICA DEL PSA TRAS LA BRAQUITERAPIA PROSTÁTICA}

La cinética del PSA tras los diferentes tratamientos activos que hoy en día existen para el tratamiento del cáncer de próstata de bajo riesgo varía considerablemente. Mientras tras la prostatectomía radical el PSA suele descender a niveles indetectables tras la cirugía, en la radioterapia externa existe un descenso progresivo del PSA hasta unos niveles variables, normalmente por debajo de $1 \mathrm{ng} / \mathrm{ml}$. En el caso de la braquiterapia nos encontramos con un descenso global más importante durante los primeros 12 meses, existiendo posteriormente una disminución menos rápida durante los 12-24 meses. Los pacientes con niveles de PSA inferiores a $1 \mathrm{ng} / \mathrm{ml}$ a los 12 meses tienen una tasa de fracaso bioquímico inferior a los que presentan PSA superior a $1 \mathrm{ng} / \mathrm{ml}$ de forma estadísticamente significativa (13).

En el caso de los pacientes sometidos a braquiterapia con implantes permanentes nos encontramos hasta en un $30-50 \%$ de los casos con ascensos del PSA que posteriormente desciende hasta los niveles de nadir e incluso por debajo (8). Este fenómeno es conocido en la literatura como PSA bounce o espículas de PSA.

\section{PSA bounce}

Estas elevaciones de PSA o PSA bounce, se presentan normalmente entre los 18 meses y los 4 años tras el implante, siendo, habitualmente, más precoces que las recidivas bioquímicas. Se ha investigado la utilidad del tiempo de doblaje de PSA como factor que diferencie entre un bounce y una recidiva bioquímica, no existiendo diferencias estadísticamente significativas entre los tiempos de doblaje de PSA de los pacientes con PSA bounce respecto a los pacientes que presentan recidiva bioquímica (18). Se caracterizan por un ascenso del PSA y un retorno a los valores del nadir o por debajo de éstos. Sin embargo, existen múltiples definiciones en cuanto a los valores a manejar en la definición de PSA bounce. Diferentes definiciones son: un incremento superior al $15 \%$ por encima del nadir seguido por un descenso hasta los niveles de éste o inferiores (9), un ascenso de al menos $0.2 \mathrm{ng} / \mathrm{ml}$ seguido por un descenso "duradero" (10), un ascenso de $0.4 \mathrm{ng} / \mathrm{ml}$ seguido de un descenso de cualquier magnitud (11), un ascenso de al menos $0.1 \mathrm{ng} / \mathrm{ml}$ seguido por un descenso de al menos $0.1 \mathrm{ng} / \mathrm{ml}$ (12) o un ascenso de al menos $0.2 \mathrm{ng} / \mathrm{ml}$ seguido por un descenso hasta los niveles del nadir o inferior a éstos (8).

La etiología del PSA bounce es controvertida. Se ha invocado la presencia de prostatitis, bacteriana o rádica, en el origen de estas espículas de PSA $(12,14)$. Incluso varios estudios demuestran ascensos transitorios de PSA en pacientes implantados tras la eyaculación, la instrumentación de la vía urinaria y la práctica del ciclismo (15 - 17). Sin embargo, estos ascensos de PSA tendrían una magnitud superior a la del PSA bounce "idiopático" (9).

Se han identificado diferentes variables predictoras de la presencia de PSA bounce. El bounce 
es más frecuente en los pacientes jóvenes $(8,10)$ y en los pacientes implantados con $1^{125}$ frente a los implantados con $\mathrm{Pd}^{103}(10)$. En un análisis multivariante, Merrick y cols. (10), encontraron que son variables predictoras independientes la edad del paciente, el estadio clínico, el primer PSA post-implante y el $V_{150}$.

Existen diversas aproximaciones a la pregunta si la presencia de una elevación de PSA tiene implicaciones pronósticas desde el punto de vista de supervivencia libre de enfermedad bioquímica. Patel y cols. (8) en un estudio retrospectivo de 295 pacientes de bajo riesgo y riesgo intermedio, tratados con braquiterapia en monoterapia, encuentran que la presencia de un PSA bounce predice una mejor supervivencia libre de enfermedad, siendo, en un análisis multivariante, únicamente la edad (pacientes menores de 65 años) un factor predictivo de presencia de este PSA bounce. En otro artículo, Critz y cols. (12) no encuentran diferencias significativas en cuanto a la recidiva bioquímica entre aquellos pacientes que han presentado un PSA bounce y los que no, pero en su serie la presencia de PSA bounce no es un factor protector contra la recidiva bioquímica.

\section{CRITERIOS DIAGNÓSTICOS DE RECIDIVA BIO- QUIMICA TRAS BRAQUITERAPIA}

La especial cinética del PSA tras un tratamiento con braquiterapia hace complicado la definición de recidiva bioquímica. Hasta el momento no existe ninguna definición de recidiva bioquímica especialmente dirigida a los pacientes tratados con braquiterapia prostática.

En pacientes tratados con radioterapia externa la existencia de diferentes definiciones de recidiva bioquímica implicaba una variabilidad que hacía imposible la comparación entre series (19). En este contexto en 1997 se publican las Consensus Guidelines for PSA Following Radiation Therapy (20), comúnmente conocidas como la definición ASTRO, convirtiéndose en el standard para la definición de recidiva bioquímica tras radioterapia. Según la ASTRO, se define la recidiva bioquímica tras radioterapia tres ascensos consecutivos de PSA separados por periodos de 6 meses, siendo la fecha de recidiva el punto medio entre el nadir y el primer ascenso de PSA. Esta definición de recidiva bioquímica ha recibido numerosas críticas, ya sea por la existencia de definiciones con mejor sensibilidad y especificidad, o por el retraso de la fecha de recidiva, fenómeno conocido como back-dating.

En un artículo de Thames y cols. se comparan más de 100 definiciones de recidiva bioquímica con la definición ASTRO, encontrándose varias definiciones con una mejor sensibilidad y especificidad en referencia al fallo clínico (21). En una posterior revisión de los datos, estudiando una cohorte de 4839 pacientes de 9 instituciones diferentes con cánceres de próstata T1-T2 tratados con radioterapia externa sin manipulación hormonal alguna, se encuentran que las definiciones con mejor sensibilidad y especificidad respecto a la definición ASTRO son: PSA superior al presente nadir en $3 \mathrm{ng} / \mathrm{ml}$ sin back-dating, PSA superior al nadir absoluto en $2 \mathrm{ng} / \mathrm{ml} \sin$ back-dating y dos ascensos consecutivos de al menos $0.5 \mathrm{ng} / \mathrm{ml}$ con back-dating (22).

Respecto al fenómeno del back-dating, encontramos que si se aplica esta práctica a las series quirúrgicas se modifican sustancialmente los resultados en cuanto a supervivencia libre de enfermedad bioquímica se refiere. Si aplicamos el criterio diagnóstico de la ASTRO a la serie del John Hopkins las supervivencias a 5,10 y 15 años pasan del $85 \%$, $77 \%$ y $68 \%$ respectivamente al $90 \%$, $90 \%$ y $90 \%$, siendo todos los fracasos precoces, existiendo un mínimo porcentaje de fracasos tardíos (23).

\section{METODOLOGÍA DIAGNÓSTICA ANTE LA RE- CIDIVA BIOQUÍMICA TRAS BRAQUITERAPIA PROSTÁTICA}

Existe una total ausencia de evidencia científica en la literatura en cuanto a cómo se debe abordar el problema de la recidiva bioquímica tras braquiterapia prostática. Esta falta de evidencia se ve incrementada por la especial evolución del PSA tras el implante, la variabilidad en la definición del PSA bounce y la mejorable definición de recidiva bioquímica propuesta por la ASTRO.

Ante esta situación, al inicio de nuestro programa de tratamiento del cáncer de próstata de bajo riesgo con braquiterapia nuestro grupo decidió adoptar la definición de recidiva de la ASTRO al igual que grupos internacionales de amplia experiencia $(5,24)$, con la intención de poder comparar nuestros resultados con las series de referencia.

En el protocolo de nuestro grupo, una vez diagnosticada la recidiva bioquímica según los criterios ASTRO, realizamos una biopsia prostática transrectal ecodirigida, obteniendo un mínimo de 5 cilindros de cada lóbulo en la actualidad. Si la biopsia es negativa, realizamos una monitorización del PSA y repetimos la biopsia en pacientes jóvenes tributarios de un tratamiento de rescate; en pacientes con edades superiores a los $70-75$ años realizamos observación en espera de la evolución del PSA para 
poder determinar su cinética, decidiendo la instauración de tratamiento hormonal en función, en la primera época, de los niveles de PSA y, en la actualidad, del tiempo de doblaje de PSA. A pesar del escaso rendimiento diagnóstico que se obtiene, decidimos realizar un reestadiaje, especialmente en pacientes jóvenes a los que se debe ofrecer una terapia de rescate.

En la actualidad disponemos de diferentes tratamientos de rescate para la recidiva bioquímica tras la braquiterapia prostática: prostatectomía radical, crioterapia prostática y braquiterapia con un implante temporal (HDR). El tratamiento siempre ha de ser individualizado, comentando con el paciente los riesgos y beneficios del mismo, teniendo muy en cuenta el entorno clínico del paciente y de la enfermedad que padece.

\section{CONCLUSIONES}

El importante porcentaje de cánceres de próstata de bajo riesgo diagnosticados en los últimos años ha propiciado el aumento de terapéuticas mínimamente invasivas tales como la braquiterapia.

El aumento en el número de tratamiento con braquiterapia realizado ha traído con sigo un nuevo escenario clínico, el de la recidiva bioquímica tras la braquiterapia prostática.

Existe una total ausencia de evidencia científica en la literatura de cómo enfocar esta nueva situación. La especial cinética de PSA tras el implante, con el fenómeno del PSA bounce y la variabilidad existente en su definición, junto con la mejorable definición de recidiva bioquímica propuesta por la ASTRO, dificultan la comparación de las series y el desarrollo de unas guías clínicas con recomendaciones de cómo actuar ante esta situación.

Nuestro grupo, al inicio del programa de braquiterapia prostática, optó por la definición ASTRO con miras a poder comparar nuestros resultados con las grandes series de la literatura. La metodología que seguimos tras el diagnóstico de recidiva es el fruto de la reflexión y el intercambio de experiencias entre Urólogos y Radioterapeutas dada la total ausencia de evidencia científica en la literatura en la que soportarse, utilizando muchas veces aproximaciones desde la metodología seguida tras la recidiva en pacientes tratados con radioterapia externa.

En esta situación es necesaria la creación de un grupo de trabajo multidisciplinar y multicéntrico para desarrollar un documento de consenso sobre el que los diferentes grupos que realizan braquiterapia prostática como tratamiento del cáncer de próstata puedan basar sus decisiones.

\section{BIBLIOGRAFÍA y LECTURAS RECOMENDADAS ( ${ }^{*}$ lectura de interés $y^{* *}$ lectura fundamental)}

1. JEMAL, A.; MURRAY, T.; WARD, E. y cols.: "Cancer statistics 2005". Cancer J. Clin., 55: 10, 2005.

2. BORRÁS, J.M.; BORRAS, J.; BOSCH, F.X. y cols.: "Càncer Catalunya 2001". Institut Català d'Oncologia, Departament de Sanitat i Seguretat Social, 2001.

**3. D'AMICO, A.V.; WHITTINGTON, R.; MALKOWICZ, S.B. y cols.: "Biochemical outcome after radical prostatectomy, external beam radiation therapy, or interstitial radiation therapy for localized prostate cancer". JAMA, 280: 969, 1998.

4. COOPERBERG, M.B.; LUBECK, D.P.; MEHTA, S.S. y cols.: "Time trends in clinical risk stratification for prostate cancer: implications for outcome (data from CAPSURE)". J. Urol., 170: 21, 2003.

**5. GRIMM, P.D.; BLASKO, J.C.; SYLVESTER, J.E. y cols.: "10-year biochemical (prostate-specific antigen) control of prostate cancer with I125 brachytherapy". Int. J. Radiation Oncology Biol. Phys., 51: 31, 2001.

**6. RAGDE, H.; KORB, L.J.; ELGAMAL, A. y cols.: "Modern prostate brachytherapy prostate specific antigen results in 219 patients with up to 12 years of observed follow-up". Cancer, 89: 135, 2000.

7. COOPERBERG, M.B.; GROSSFELD, G.D.; LUBECK, D.P. y cols.: "National practice patterns and time trends in androgen ablation for localized prostate cancer". J. Natl. Cancer Inst., 95: 981, 2003.

*8. PATEL, C.; ELSHAIKH, M.; ANGERMEIER, K. y cols.: "PSA bounce predicts early success in patients with permanent iodine- 125 prostate implant". Urology, 63: 110, 2004.

*9. DAS, P.; CHEN, M.H.; VALENTINE, K. y cols.: "Using the magnitude of PSA bounce alter MRIguided prostate brachytherapy to distinguish recurrence, benign precipitating factors and idiopathic bounce". Int. J. Radiation Oncology Biol. Phys., 54: 698, 2002.

*10. MERRICK, G.S.; BUTLER, W.M.; WALLNER, K.E. y cols.: "Prostate-specific antigen spikes after permanent prostate brachytherapy". Int. J. Radiation Oncology Biol. Phys., 54: 450, 2002.

11. HANLON, A.L.; PINOVER, W.H.; HORWITZ, E.M. y cols.: "Patterns and fate of PSA bouncing 
following 3D-CRT". Int. J. Radiation Oncology Biol. Phys., 50: 845, 2001.

*12. CRITZ, F.A.; WILLIAMS, W.H.; BENTON, J.B. y cols.: "Prostate specific antigen bounce after radioactive seed implantation followed by external beam radiation for prostate cancer". J. Urology, 163: 1085, 2000.

13. IANNUZZI, C.M.; STOCK, R.G.; STONE, N.N.: "PSA kinetics following 125-I radioactive seed implantation in the treatment of T1-T2 prostate cancer". Radiat. Oncol. Investig., 7: 30, 1999.

14. CAVANAGH, W.; BLASKO, J.C.; GRIMM, P.D. y cols.: "Transient elevation of serum prostatespecific antigen following 125I/103Pd brachytherapy for localized prostate cancer". Semin. Urol. Oncol., 18: 160, 2000.

15. TCHETGEN, M.B.; SONG, J.T.; STRAWDERMAN, M. y cols.: "Ejaculation increases the serum prostate-specific antigen concentration". Urology, 47: 511, 1996.

16. DEW, T.; COCKER, C.; SAADEH, F. y cols.: "Influence of investigative and operative procedures on serum prostate-specific antigen”. Ann Clin. Biochem., 36: 340, 1999.

17. CRAWFORD, E.D. III.; MACKENZIE, S.H.; SAFFORD, H.R. y cols.: "The effect of bicycle riding on serum prostate specific antigen levels". J. Urology, 156: 103, 1996.

*18. CIEZKI, J.P.; REDDY, C.A.; GARCIA, J. y cols.: "PSA kinetics after prostate brachytherapy: PSA bounce phenomenon and its implications for PSA doubling time". Int. J. Radiation Oncology Biol. Phys., 64: 512, 2006.
19. HORWITZ, E.M.; VICINI, F.A.; VIAJA, E.L. y cols.: "Assessing the variability outcome for patients treated with localized prostate irradiation using different definitions of biochemical control”. Int. J. Radiation Oncology Biol. Phys., 36: 565, 1996.

20. AMERICAN SOCIETY FOR THERAPEUTIC RADIOLOGY AND ONCOLOGY CONSENSUS PANEL: "Consensus statement: guidelines for PSA following radiation therapy". Int. J. Radiation Oncology Biol. Phys., 37: 1035, 1997.

21. THAMES, H.; SUBAN, D.; LEVY, L. y cols.: "Comparison of alternative biochemical failure definitions base on clinical outcome in 4839 prostate cancer patients treated by external beam radiotherapy between 1986 and 1995". Int. J. Radiation Oncology Biol. Phys., 57: 929, 2003.

22. HORWITZ, E.M.; THAMES, H.D.; SUBAN, D.A. y cols.: "Definitions of biochemical failure that best predict clinical failure in patients with prostate cancer treated with external beam radiation alone: a multi-institutional pooled analysis". J. Urology, 173: 797, 2005.

23. GRETZER, M.B.; TROCK, B.J.; HAN, M. y cols.: "A critical analysis of the interpretation of biochemical failure in surgically treated patients using the american society for therapeutic radiation and oncology criteria”. J. Urology, 168: 1419, 2002.

24. STONE, N.N.; STOCK, R.G.; UNGER, P.: "Intermediate term biochemical-free progression and local control following 125iodine brachytherapy for prostate cancer". J. Urology, 173: 803, 2005. 